\title{
X-ray flashes or soft gamma-ray bursts?
}

\section{The case of the likely distant XRF 040912}

\author{
G. Stratta ${ }^{1}$, S. Basa ${ }^{2}$, N. Butler ${ }^{3}$, J. L. Atteia ${ }^{1}$, B. Gendre ${ }^{4}$, A. Pélangeon ${ }^{1}$, F. Malacrino ${ }^{1}$, Y. Mellier ${ }^{5}$, D. A. Kann ${ }^{6}$, \\ S. Klose ${ }^{6}$, A. Zeh ${ }^{6}$, N. Masetti ${ }^{7}$, E. Palazzi ${ }^{7}$, J. Gorosabel ${ }^{8}$, A. J. Castro-Tirado ${ }^{8}$, A. de Ugarte Postigo ${ }^{8}$, M. Jelinek ${ }^{8}$, \\ J. Cepa ${ }^{9}$, H. Castañeda ${ }^{9}$, D. Martínez-Delgado ${ }^{9}$, M. Boër ${ }^{10}$, J. Braga ${ }^{11}$, G. Crew ${ }^{12}$, T. Q. Donaghy ${ }^{13}$, J.-P. Dezalay ${ }^{14}$, \\ J. Doty ${ }^{12}$, E. E. Fenimore ${ }^{15}$, M. Galassi ${ }^{15}$, C. Graziani ${ }^{13}$, J. G. Jernigan ${ }^{16}$, N. Kawai ${ }^{17,18}$, D. Q. Lamb ${ }^{13}$, A. Levine ${ }^{12}$, \\ R. K. Manchanda ${ }^{19}$, F. Martel ${ }^{12}$, M. Matsuoka ${ }^{20}$, Y. Nakagawa ${ }^{22}$, J.-F. Olive ${ }^{14}$, G. Pizzichini ${ }^{7}$, G. Prigozhin ${ }^{12}$, \\ G. Ricker ${ }^{12}$, T. Sakamoto ${ }^{25}$, Y. Shirasaki ${ }^{18,21}$, S. Sugita ${ }^{22}$, M. Suzuki ${ }^{18}$, K. Takagishi ${ }^{23}$, T. Tamagawa ${ }^{18}$, \\ R. Vanderspek ${ }^{12}$, J. Villasenor ${ }^{12}$, S. E. Woosley ${ }^{24}$, M. Yamauchi ${ }^{23}$, and A. Yoshida ${ }^{18,22}$
}

(Affiliations can be found after the references)

Received 14 June 2006 / Accepted 28 September 2006

\begin{abstract}
Context. The origin of X-ray Flashes (XRFs) is still a mystery and several models have been proposed. To disentangle among these models, an important observational tool is the measure of the XRF distance scale, so far available only for a few of them.

Aims. In this work, we present a multi-wavelength study of XRF 040912, aimed at measuring its distance scale and the intrinsic burst properties. Methods. We performed a detailed spectral and temporal analysis of both the prompt and the afterglow emission and we estimated the distance scale of the likely host galaxy. We then used the currently available sample of XRFs with known distance to discuss the connection between XRFs and classical Gamma-ray Bursts (GRBs).

Results. We found that the prompt emission properties unambiguously identify this burst as an XRF, with an observed peak energy of $E_{\mathrm{p}}=$ $17 \pm 13 \mathrm{keV}$ and a burst fluence ratio $S_{2-30 \mathrm{keV}} / S_{30-400 \mathrm{keV}}>1$. A non-fading optical source with $R \sim 24$ mag and with an apparently extended morphology is spatially consistent with the X-ray afterglow, likely the host galaxy. XRF 040912 is a very dark burst since no afterglow optical counterpart is detected down to $R>25 \mathrm{mag}$ ( $3 \sigma$ limiting magnitude) at $13.6 \mathrm{~h}$ after the burst. The host galaxy spectrum detected from $3800 \AA$ to $10000 \AA$, shows a single emission line at $9552 \AA$. The lack of any other strong emission lines blue-ward of the detected one and the absence of the Ly $\alpha$ cut-off down to $3800 \AA$ are consistent with the hypothesis of the [OII] line at redshift $z=1.563 \pm 0.001$. The intrinsic spectral properties rank this XRF among the soft GRBs in the $E_{\text {peak }}-E_{\text {iso }}$ diagram. Similar results were obtained for most XRFs at known redshift. Only XRF 060218 and XRF 020903 represent a good example of instrinsic XRF $(i-\mathrm{XRF})$ and are possibly associated with a different progenitor population. This scenario may call for a new definition of XRFs.
\end{abstract}

Key words. gamma rays: bursts

\section{Introduction}

X-ray Flashes (XRFs; Heise et al. 2001; Kippen et al. 2001) are extra-galactic transient X-ray sources with spatial distribution, spectral and temporal characteristics similar to long duration Gamma-Ray Bursts (GRBs). The remarkable property that distinguishes XRFs from GRBs is that their $v F_{v}$ prompt emission spectrum peaks at energies which are observed to be typically one order of magnitude lower than the observed peak energies of GRBs. XRFs are empirically defined by a greater fluence (time integrated flux) in the X-ray band $(2-30 \mathrm{keV})$ than in the gammaray band (30-400 keV). Along with the intermediate class of bursts called X-ray Rich bursts (XRRs), XRFs and GRBs form a continuum in the observed peak energy versus the $2-400 \mathrm{keV}$ fluence plane, defining the well known hardness-intensity relation for which soft bursts are also faint, in the observer reference frame (Barraud et al. 2003; Sakamoto et al. 2005). The XRF optical and X-ray afterglow flux distribution at about 0.5 days from the burst trigger is comparable to the one observed for GRB afterglows (D'Alessio et al. 2005). Despite the lack of any clear evidence of achromatic temporal breaks so far, XRF afterglow temporal decay indices are on average consistent with those commonly observed for GRB and XRR afterglows before or after the temporal break (D'Alessio et al. 2005).

As soon as they were discovered, XRFs were thought to be high redshift $(z \gtrsim 5)$ GRBs, so that their softness and faintness were an effect of the cosmological distance (Heise et al. 2003). However, the absence of any evidence of time dilation in the temporal properties of XRFs with respect to nearby GRBs motivated other explanations. The origin of XRFs has been explored in the context of the fireball model, where bursts fainter and softer than GRBs can be produced by: $i$ ) high baryon-loaded fireballs, assuming that both the prompt emission and the afterglow are produced by external shocks (“dirty fireball", e.g. Dermer et al. 1999); ii) low-efficiency internal shocks due to low-velocity contrast between the high velocity colliding shells (e.g. Mochkovitch et al. 2004; Barraud et al. 2005). Alternatively, it has been proposed that XRFs and GRBs are indeed the same phenomenon. In this case, their diversity is associated to the aperture of the jet opening angle (Jet Variable Opening Angle model) or to the observer viewing angle from the jet axis, for a structured energy density distribution within the jet (Structured Jet model; see Lamb et al. 2005, for a 
review). In the so-called "off-axis" GRB model, the observer line-of-sight is outside the jet cone. In this case, the prompt emission is detectable only when the relativistic beaming angle is large enough to enter the line-of-sight (e.g. Yamazaki et al. 2002). Alternatively, an XRF is observed in a sideway vision at the moment when the jet is breaking out from a hot cocoon surrounding the GRB (e.g. Mészáros et al. 2002).

An important observational tool to disentangle the proposed models is the measure of the distance scale of XRFs and therefore the intrinsic properties of the burst. Among 35 XRFs observed so far (from BeppoSAX and HETE-2 archives, see D'Alessio et al. 2005; and Sakamoto et al. 2005; and from J. Greiner's web page ${ }^{1}$ from September 2003 to August 2006), the ones at known redshift are XRF 020903 at $z=0.25 \pm 0.01$ (Soderberg et al. 2004; see also Bersier et al. 2006), XRF 030429 at $z=2.658 \pm 0.004$ (Jakobsson et al. 2004), XRF 030528 at $z=0.782 \pm 0.001$ (Rau et al. 2005), XRF 040701 at $z=0.2146$ (Kelson et al. 2004), XRF 050416A at $z=0.6528 \pm 0.0002$ (Soderberg et al. 2006), XRF 050824 at $z=0.83$ (Crew et al. 2005; Fynbo et al. 2005) and XRF 060218 at $z=0.03342$ (Pian et al. 2006). Recently, GRB 981226, detected with the BeppoSAX satellite, has been reanalyzed by D'Alessio et al. (2005) and classified as an XRF, at $z=1.11 \pm 0.06$ (Christensen et al. 2005). As already pointed out by Rau et al. (2005), some XRFs (e.g. XRF 030528, XRF 030429) would have been detected as XRR bursts or GRBs if observed in their rest frame, possibly suggesting that some XRFs do not require a different emission model than for GRBs.

In this paper we present and discuss the results obtained from a multi-wavelength analysis of XRF 040912 aimed at measuring its distance scale and the intrinsic burst properties. In Sect. 2 we provide a brief review of the follow-up campaign of XRF 040912. In Sect. 3 we present the data reduced in this work and the performed analysis. In Sect. 4 we present the results obtained from our analysis while in Sect. 5 we discuss the dark nature of this burst and we compare the intrinsic properties of XRF 040912 with those observed for other XRFs and GRBs. In Sect. 6 we discuss the implications of our observations for the nature of X-ray flashes.

\section{Observations}

XRF 040912 was a soft, long burst discovered with the WideField X-Ray Monitor (WXM) and the French Gamma Telescope (FREGATE) on board the High Energy Transient Explorer-2 (HETE-2) on 2004, September 12.592 UT (Butler et al. 2004a). The $7^{\prime}$ WXM error-box was centered at RA $=23^{\mathrm{h}} 56^{\mathrm{m}} 53^{\mathrm{s}} .52$ and Dec $=-01^{\circ} 00^{\prime} 03^{\prime \prime}$. 6 (J2000.0). Optical follow-up started $1.8 \mathrm{~h}$ after the burst (Ogura et al. 2004) with several ground based telescopes but, despite very deep observations, no evidence of a transient source was found. The Chandra Observatory targeted the WXM error-box in two epochs, from 3.32 to 3.57 days and from 8.86 to 9.12 days after the burst (Butler et al. 2004b). Among 22 X-ray sources found, only one (CXOU J235642.9005520 , hereafter $\mathrm{S} 1$ ) showed a significantly fading behavior. Two $R$-band observations of the HETE- 2 WXM error region were performed in two epochs with the $27^{\prime} \times 27^{\prime}$ FOV IMACS camera on the Magellan $6.5 \mathrm{~m}$ Baade Telescope at Las Campanas Observatory (Butler et al. 2004c), with $3 \times 180$ s exposures at $13.57 \mathrm{~h}$ after the burst and $2 \times 300 \mathrm{~s}$ exposures at $38.65 \mathrm{~h}$ after the burst (mean observing time). From these observations a persistent optical source spatially consistent with the fading

\footnotetext{
${ }^{1}$ http://www.mpe.mpg.de/ jcg/grbgen.html
}

X-ray source S1 was found, while an apparently fading optical source was found positionally consistent with the Chandra source CXOU J235656.4-005839 (hereafter S2) that was nonfading in X-rays (Butler et al. 2004c). Further optical images have been acquired (Gorosabel et al. 2004) starting $7.25 \mathrm{~h}$ after the event with the $4.2 \mathrm{~m}$ William Herschel Telescope (WHT) equipped with the Prime Focus Camera (PFC) at La Palma $\left(16^{\prime} \times 16^{\prime} \mathrm{FOV}\right)$ and 0.5 days after the burst with the Sierra Nevada Observatory (OSN) $1.5 \mathrm{~m}$ Telescope. Internal comparison performed between a sequence of $V$-band images, within $7.7 \mathrm{~h}$ and 3.5 days after the burst, did not reveal any variability in the entire HETE-2 error-box (Gorosabel et al. 2004).

From the Tautenburg $1.34 \mathrm{~m}$ Schmidt telescope (TLS) $B V R_{\mathrm{C}} I_{\mathrm{C}}$ observations were obtained between 5 and $11 \mathrm{~h}$ after the burst. In addition, $R_{\mathrm{C}}$ and $I_{\mathrm{C}}$ observations and then $I_{\mathrm{C}}$ observations were obtained one day later and three days after the burst, respectively (Klose et al. 2004), with no evidence of transient source. On 2004 Sept. 23.24 UT (10.6 days after the burst), Very Large Array follow-up observations at $8.46 \mathrm{GHz}$ did not reveal any radio counterpart of the $\mathrm{X}$-ray afterglow candidate down to a $3 \sigma$ limit of $120 \mu \mathrm{Jy}$ (Soderberg et al. 2004). On October 5.42 UT and on November 18.35 UT (22.8 and 66.76 days after the burst) we observed the X-ray afterglow candidate with the widefield imager MegaCam (36 2048 ×4612 pixel CCDs, 1 square degree FOV), mounted on the $3.6 \mathrm{~m}$ Canadian-French-Hawaii Telescope (CFHT), with $i^{\prime}$-band $2 \times 860 \mathrm{~s}$ exposures, in order to look for any SN emission (Stratta et al. 2004a). We obtained further I-band observations with the $8.2 \mathrm{~m}$ Very Large Telescope (VLT) equipped with Focal Reducer Spectrometer FORS2 on the 30th of September and on the 4th of October 2005. Imaging and spectroscopy of the likely host galaxy were performed under the Director's Discretionary Time Proposal 275.A-5041.

\section{Data reduction and analysis}

Spectra and light curves of the prompt emission were extracted from the HETE-2/WXM (2-25 keV) and HETE-2/FREGATE (6-400 keV) data. The spectral analysis was performed with a joint WXM and FREGATE data fit. The spectra have been integrated over $146.8 \mathrm{~s}$, that is from $T_{0}-12.7 \mathrm{~s}$ to $T_{0}+134.1 \mathrm{~s}$, where $T_{0}$ is the HETE-2 trigger time. The joint spectral fits WXM/FREGATE have been performed within the 5-200 keV energy range, using the XSPEC v.11.2.0 software package.

Spectral analysis was performed for each Chandra Target of Opportunity observations (ToO), with integration time of $18.2 \mathrm{ks}$ and $19.5 \mathrm{ks}$ respectively, for the two sources that showed a fading behavior (S1 and S2, see Sect. 2). We reprocessed the data using the latest packages available (CIAO v 3.3.0.1 and caldb v 3.2.1) and the standard procedures described on the CIAO webpage.

Astrometry and photometry of the Magellan, TLS, WHT, OSN and VLT/FORS2 images have been performed following standard procedures. Magnitudes have been calibrated against the USNO stars of Henden et al. (2004). Photometry was performed using the SExtractor software (Bertin \& Arnouts 1996). In order to check for flux variability, the two epochs Magellan images have been subtracted with the dedicated package ISIS (Alard 2000). For the CFHT/MEGACAM images, flat-field and bias correction were performed with the Elixir pre-processing tool at the CFHT. Quality assessment of individual and stacked images, astrometric calibration, image re-centering and the full stacked process, were performed at the Terapix astronomical data reduction center. Unfortunately, the poor quality of the second CFHT/MEGACAM epoch observations (see Sect. 2) prevented us to check for the presence of any supernova component 


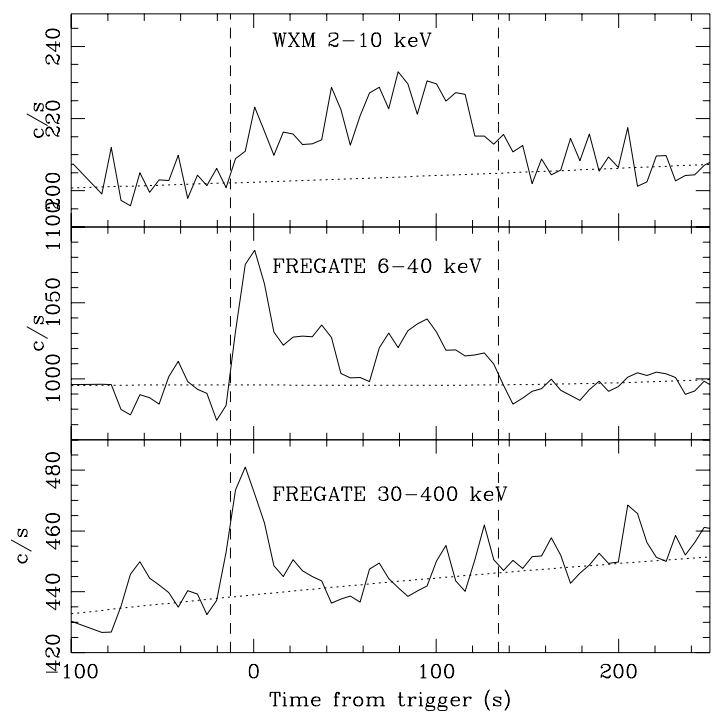

Fig. 1. The count rate evolution over the time after the trigger of the prompt emission (temporal resolution of $4 \mathrm{~s}$ per bin). The two vertical dashed lines mark the temporal interval in which we extracted the energy spectrum of the burst from the WXM and FREGATE data. The dotted line is a fit of the background count rate evolution with time.

through flux variability. VLT/FORS2 long slit spectroscopy has been performed with $4 \times 1100$ s exposures using the G150I grism, covering the spectral range of $3500 \div 10000 \AA$. Spectra have been reduced by using an automatic pipeline especially developed in the framework of The SuperNova Legacy Survey (Basa et al. in preparation).

\section{Results}

\subsection{Prompt emission}

The count rate light curve of the prompt emission of XRF 040912 shows a long and soft burst (Fig. 1). We computed the burst durations, represented with the $T_{90}$ and $T_{50}$ parameters, in different energy bands (Table 1, quoted errors are at $1 \sigma$ level). The $5-200 \mathrm{keV}$ burst spectrum is adequately fit by a simple power-law model or by a cut-off power-law model (Table 2). The Band model (Band et al. 1993) does not allow to well constrain the parameters. We found a minimum $\chi^{2}$ by fixing the high energy photon index $\Gamma_{2}$ to -3.0 (Table 2). Fixing the low energy photon index $\Gamma_{1}$ to the best fit value obtained from the cut-off power-law model and $\Gamma_{2}$ to the typical value observed for GRBs of -2.3 (Sakamoto et al. 2005), we obtain a peak energy of $E_{\text {peak }}=17 \pm 13 \mathrm{keV}$ and X-ray and gamma-ray fluences of $S_{2-30 \mathrm{keV}}=9.5 \times 10^{-7} \mathrm{erg} \mathrm{cm}^{-2}$ and $S_{30-400 \mathrm{keV}}=7.4 \times 10^{-7} \mathrm{erg} \mathrm{cm}^{-2}$. The low value of the derived peak energy and the fluence ratio $S_{2-30 \mathrm{keV}} / S_{30-400 \mathrm{keV}}>1$ unambiguously identify this burst as an X-ray flash.

\subsection{Afterglow identification}

From refined photometric analysis of the Magellan Telescope images, we find that the fading behavior of the optical source associated with S2 (Butler et al. 2004c, see Sect. 2) is not real, being an artifact due to bad calibration that brought to a flux overestimation of this particular source during the first epoch. We further confirm this result also from the TLS $R$-band observations, from which we detect the source in three epochs $(0.270$, 0.431 and 1.353 days after the burst event) with flux constant
Table 1. Burst duration at different energy ranges of XRF 040912.

\begin{tabular}{llll}
\hline \hline Instrument & $\Delta E[\mathrm{keV}]$ & $T_{90}[\mathrm{~s}]$ & $T_{50}[\mathrm{~s}]$ \\
\hline HETE-2/WXM & $2-25$ & $143 \pm 10$ & $70.0 \pm 9.5$ \\
HETE-2/FREGATE & $6-40$ & $127 \pm 13$ & $80 \pm 5$ \\
& $30-400$ & $104 \pm 26$ & $53 \pm 19$ \\
\hline
\end{tabular}

with time. From the Magellan image subtraction, we find no evidence of any residual for both afterglow candidates S1 and S2 down to $R>25.3 \mathrm{mag}$ ( $3 \sigma$ limiting magnitude). The afterglow identification is therefore addressed to the $\mathrm{X}$-rays observations.

The Chandra source S1 (see Sect. 2) is the only one that showed a statistically significant fading behavior in the X-rays. The extracted $0.2-10.0 \mathrm{keV}$ spectra have been fitted with a simple power-law model plus an absorption component. During the first $\mathrm{ToO}$, the spectral properties of this source show an equivalent neutral hydrogen column density $N_{\mathrm{H}}$ consistent with the Galactic value of $3.7 \times 10^{20} \mathrm{~cm}^{-2}$ (Dickey \& Lockman 1990), an energy spectral index of $\alpha=1.4_{-0.9}^{+1.0}$ and a $2-10 \mathrm{keV}$ flux of $(7.1 \pm 1.5) \times 10^{-15} \mathrm{erg} \mathrm{cm}^{-2} \mathrm{~s}^{-1}$. These values are consistent with those estimated for other X-ray afterglows observed at similar epochs (e.g. De Pasquale et al. 2006). During the second $\mathrm{ToO}$ the statistics is too poor to constrain the spectral parameters. Assuming the best fit model found in the first ToO and leaving the normalization free to vary, the $2-10 \mathrm{keV}$ flux is $(2.9 \pm 1.0) \times 10^{-15} \mathrm{erg} \mathrm{cm}^{-2} \mathrm{~s}^{-1}$ implies a decay index of $\delta_{\mathrm{X}}=-1.0 \pm 0.6$ consistent with the previous findings of Ford et al. (2004, quoted errors are at $90 \%$ confidence level).

For the source S2, the spectral model is poorly constrained due to the faintness of this source. Assuming the same spectral parameters derived for S1, we found a flux of $(1.3 \pm 0.5) \times$ $10^{-15} \mathrm{erg} \mathrm{s}^{-1} \mathrm{~cm}^{-2}$ and $(1.0 \pm 0.6) \times 10^{-15} \mathrm{erg} \mathrm{s}^{-1} \mathrm{~cm}^{-2}$ in the first and second $\mathrm{ToO}$ respectively. The corresponding decay index is then $\delta_{\mathrm{X}}=-0.3_{-1.3}^{+1.0}(90 \%$ confidence level), confirming previous analysis (Ford et al. 2004) that classified this source as consistent with being constant in flux with time.

We conclude that, despite the unknown probability of finding a transient source within $7^{\prime}$ error region with the observed decay index, since $\mathrm{S} 1$ is the only Chandra source among 22 detected in the HETE-2 error-box that shows a statistically significant fading behavior in X-rays and both its spectral and temporal properties are consistent with the typical X-ray afterglows observed at similar epochs (few days after the burst), this source provides convincing evidence to be the X-ray afterglow of XRF 040912.

We find no fading optical counterpart for this source from a set of several optical images taken at different telescopes and at different epochs (see Table 3), ranking this burst among the most darkest XRF ever observed (Fig. 5).

\subsection{The likely host galaxy}

The non-fading nature of the optical source positionally consistent with the X-ray afterglow candidate and its apparently extended morphology, strongly suggest that this source is the host galaxy of XRF 040912 (Fig. 4). We computed the chance probability to find a galaxy with $R \leq 24.0$ mag within the 1 " radius error circle. Following Piro et al. (2002), we found $P=1.8 \times 10^{-2}$. This value makes the chance of association unlikely, though not negligible.

The VLT/FORS2 extracted spectrum of this source is well detected between $3800 \AA$ and $10000 \AA$, implying a robust 
Table 2. Results from WXM (5-25 keV) and FREGATE (7-200 keV) joint spectral analysis.

\begin{tabular}{|c|c|c|c|c|c|c|c|}
\hline Model & $\overline{\Gamma_{1}}$ & $\overline{\Gamma_{2}}$ & $\overline{E_{0}(\mathrm{keV})}$ & $\overline{E_{\text {peak }}(\mathrm{keV})}$ & Norm. WXM & Norm. FREGATE & $\chi_{v}^{2} /$ d.o.f. \\
\hline$\overline{\mathrm{PL}^{a}}$ & - & $-2.15_{-0.19}^{+0.22}$ & - & - & $1.56_{-0.57}^{+0.96}$ & $2.37_{-1.00}^{+1.79}$ & $1.109 / 104$ \\
\hline CPL $1^{b}$ & $-1.25_{-0.58}^{+0.83}$ & $-{ }^{-0.19}$ & $26.98_{-15.58}^{+52.85}$ & - & $0.34_{-0.25}^{+0.62}$ & $0.39_{-0.27}^{+0.64}$ & $1.035 / 103$ \\
\hline $\mathrm{CPL} 2^{c}$ & $-1.25_{-0.58}^{+0.82}$ & - & - & $20.23_{-539}^{+9.54}$ & $\left(1.14_{-053}^{+1.70}\right) 10^{-2}$ & $\left(1.53_{-0.61}^{+2.09}\right) 10^{-2}$ & $1.035 / 103$ \\
\hline Band & $-1.18_{-1.16}^{+1.23}$ & $-3.0($ fixed $)$ & $22.61_{-15.02}^{+73.53}$ & - & $\left(0.14_{-0.12}^{+5.81}\right) 10^{-2}$ & $\left(0.18_{-0.10}^{+7.38}\right) 10^{-2}$ & $1.104 / 103$ \\
\hline
\end{tabular}

${ }^{a} \mathrm{PL}=$ Power-law model; ${ }^{b} \mathrm{CPL} 1$ = Cutoff power-law model with photon index, $E_{0}$ and normalization at $1 \mathrm{keV} ;{ }^{c} \mathrm{CPL} 2=\mathrm{Cutoff}$ power-law model with photon index, $E_{\text {peak }}=(2+\alpha) E_{0}$ and normalization at $15 \mathrm{keV}$.

Table 3. Optical detections and upper limits at the position of the X-ray afterglow candidate. Magnitudes have been corrected for the Galactic reddening $(E(B-V)=0.028$, Schlegel et al. 1998).

\begin{tabular}{|c|c|c|c|}
\hline Telescope & $\begin{array}{l}\Delta T^{a} \\
\text { days }\end{array}$ & $\begin{array}{l}\text { Exp. } \\
\text { s }\end{array}$ & mag \\
\hline TLS & 0.270 & 1800 & $R_{\mathrm{C}}>22.6$ \\
\hline TLS & 0.289 & 1800 & $I_{\mathrm{C}}>21.0$ \\
\hline TLS & 0.309 & 1800 & $B>22.7$ \\
\hline WHT & 0.312 & 1455 & $B>25.0$ \\
\hline WHT & 0.325 & 900 & $V>24.4$ \\
\hline TLS & 0.328 & 1800 & $V>22.9$ \\
\hline TLS & 0.431 & 3360 & $R_{\mathrm{C}}>22.9$ \\
\hline OSN & 0.553 & 540 & $I>21.0$ \\
\hline Magellan $^{b}$ & 0.565 & 540 & $R=23.89 \pm 0.10$ \\
\hline WHT & 0.599 & 900 & $V>24.7$ \\
\hline WHT & 1.294 & 1200 & $V>24.7$ \\
\hline TLS & 1.353 & 4320 & $R_{\mathrm{C}}>23.3$ \\
\hline TLS & 1.367 & 3600 & $I_{\mathrm{C}}>21.2$ \\
\hline Magellan $^{b}$ & 1.610 & 600 & $R=24.07 \pm 0.10$ \\
\hline TLS & 3.308 & 2700 & $I_{\mathrm{C}}>21.0$ \\
\hline WHT & 3.489 & 1500 & $I>22.1$ \\
\hline CFHT & 22.8 & 1720 & $i^{\prime}=24.02 \pm 0.16$ \\
\hline CFHT & 66.7 & 1720 & $i^{\prime}>22.8$ \\
\hline VLT & 385 & 690 & $I=23.96 \pm 0.10$ \\
\hline
\end{tabular}

${ }^{a} \Delta T$ is the time from the burst event; ${ }^{b}$ from image subtraction, we find no residual down to $R>25.3$ ( $3 \sigma$ upper limit).

redshift limit of $z<2.13$ from the absence of the Ly $\alpha$ absorption due to the neutral hydrogen present along the line-of-sight. The most statistically significant feature is an emission line at the red edge of the spectrum centered at $\lambda_{\text {line }}=9552 \AA$, with signal to noise ratio of more than 10 (Fig. 3). If interpreted as the $\mathrm{H} \alpha$ ( $\lambda 6563 \AA$ ) line redshifted at $z=0.455$, then it would be difficult to explain the absence of other emission lines as the [OII] $(\lambda 3727 \AA)$ and the [OIII] $(\lambda 5007 \AA)$, expected to be observed at $5423 \AA$ and $7285 \AA$, respectively. Another interpretation, consistent both with the lack of the Ly $\alpha$ cut-off and with the absence of any other strong emission line blue-ward of the detected one, is that we are indeed observing the $[\mathrm{OII}](\lambda 3727 \AA)$ emission line, redshifted to $z=1.563 \pm 0.001$. According to this interpretation, we further identify three statistically less significant features as the $\operatorname{MgII}(\lambda 2798 \AA)$ and the FeII $(\lambda 2586 \AA)$ absorption lines and the CIII $(\lambda 1909 \AA)$ narrow emission line.

In order to estimate the metallicity and the dominant stellar population age, we fitted the spectrum with several template spectra. Templates are from the library of evolutionary stellar population synthesis models GALAXEV based on the isochrone synthesis code of Bruzual \& Charlot (2003). Due to the very low signal to noise ratio, we fit mainly the spectral continuum. Doing so, we are only sensitive to the age of the dominant population which fixes the slope and not to the metallicity. By fixing the metallicity to different values, we find that in any case the dominant stellar population age should be less than $0.2 \mathrm{Gyr}$.

From the [OII] line luminosity, we attempted to estimate the star formation rate (SFR) for this galaxy. We measure the strength of the line by a Gaussian fit performed with the IRAF ${ }^{2}$ splot package and we find $(0.57 \pm 0.04) \times 10^{-16} \mathrm{erg} \mathrm{cm}^{-2} \mathrm{~s}^{-1}$. Applying the relation from Kennicutt (1998) we find a SFR of $(6.3 \pm 0.4) M_{\odot} \mathrm{yr}^{-1}$. An alternative method to measure the SFR is through the UV $(1500 \AA \div 2800 \AA$ ) luminosity (Kennicutt 1998). The average UV flux of our spectrum is $(1.36 \pm 0.50) \times$ $10^{-19} \mathrm{erg} \mathrm{cm}^{-2} \mathrm{~s}^{-1} \AA^{-1}$ from which the inferred SFR is $(2.3 \pm$ 0.8) $M_{\odot} \mathrm{yr}^{-1}$. We note that the latter evaluation is more robust than the former one since the UV luminosity is a direct tracer of metallicity. The derived SFR values have not been corrected for a possible local extinction and therefore $(2.3 \pm 0.8) M_{\odot} \mathrm{yr}^{-1}$ should be considered as the lower limit of the real SFR.

Our findings are consistent with the typical SFR range of values $\left(0.7 \div 12.7 M_{\odot} \mathrm{yr}^{-1}\right.$, not corrected for extinction) found for GRBs (Christensen et al. 2004). Together with the age estimation obtained by $\chi^{2}$ minimization from the template spectra fitting, a coherent description of a late type galaxy with moderatehigh star formation activity is emerging, further strengthening the identification of this galaxy as the host of XRF 040912.

\section{Discussion}

\subsection{A "dark" X-ray flash}

The optical flux upper limits of XRF 040912 measured from different telescopes (Table 3) are among the faintest ever obtained (Fig. 5), ranking this burst among the "darkest" XRFs (and GRBs, see e.g. Rol et al. 2005). We note that the Galactic extinction towards this burst is small. Indeed, from Schlegel et al. (1998), the Galactic reddening is $E(B-V)=0.028$. The observed faintness of XRF 040912 cannot be ascribed only to distance since, for example, the more distant XRF 030429 shows a much brighter flux than this case. This suggests an intrinsically faint and/or rapidly fading early afterglow or a highly extinguished line-of-sight.

The X-ray afterglow flux is among the faintest ones but still comparable to other XRF X-ray afterglows observed at similar epochs (a few days after the burst event). In particular, both the flux and decay rate are very similar to those observed for the Xray afterglow of XRF 030723 , for which however a bright optical counterpart has been detected (Figs. 2 and 5). A useful piece of information comes from the likely host galaxy of XRF 040912

\footnotetext{
${ }^{2}$ IRAF (Image Reduction and Analysis Facility) is written and supported by the IRAF programming group at the National Optical Astronomy Observatories (NOAO) in Tucson, Arizona. NOAO is operated by the Association of Universities for Research in Astronomy (AURA), Inc. under cooperative agreement with the National Science Foundation. IRAF is available at http://iraf.noao. edu/
} 


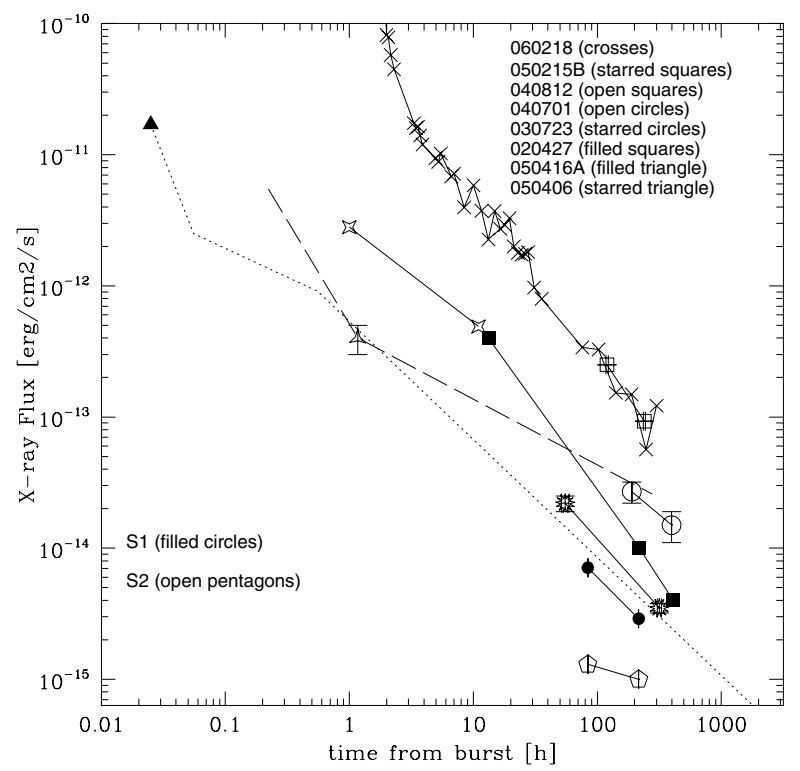

Fig. 2. X-ray afterglow light curves of a sample of XRFs for which we could trace the decaying behavior (from GCNs), and the two afterglow candidates of XRF 040912 S1 and S2 (see Sect. 2). For XRF 050416A, we plot the first measurement and we trace the decay behavior derived by Mangano et al. (2006). For XRF 050406 we plot the flux at the break and the best fit broken power law derived by Romano et al. (2006). Other data are from: Campana et al. (2006) for XRF 060218, Levan et al. (2006) for XRF 050215B, Campana et al. (2004) for XRF 040812, Fox et al. (2004) for XRF 040701, Butler et al. (2003) for XRF 030723, Fox et al. (2002) for XRF 020427. While S1 (filled circles) shows a typical temporal behavior, S2 (open pentagons) shows an anomalous decay.

that shows no evidence of reddening, with a "flat" spectral continuum (Fig. 3). A possible interpretation of these findings is that the afterglow of this XRF is intrinsically fainter with respect to other XRFs. Another explanation which we cannot exclude is that the optical emission was suppressed by dust. The lack of reddening in the host galaxy spectrum may be explained by a localized dusty environment in the vicinity of the XRF, as suggested for GRB 000210, another "dark" burst with similar characteristics (Gorosabel et al. 2003). Alternatively, the lack of reddening may be due to a weak wavelength dependence of the dust extinction law, as inferred for some GRBs and for other extragalactic objects such as AGNs (Maiolino et al. 2000; Stratta et al. 2004b; Chen et al. 2006). In the latter case, a high equivalent hydrogen column density is expected from the X-ray data analysis, while we found a value consistent with the Galactic absorption. However, the X-ray data quality is too low to exclude a large hydrogen column density value. A simple exploration of the parameter space, leaving all the model parameters (see Sect. 3.3) free to vary, indicates an extra-galactic absorption upper limit of $N_{\mathrm{H}} \leq 7.4 \times 10^{22} \mathrm{~cm}^{-2}$ (95\% confidence level) assuming a solar abundance and a cold, neutral gas. Therefore, although we cannot exclude the presence of a large amount of neutral hydrogen, we consider that this possibility is not strongly supported by the observations.

\subsection{Energetics}

The estimation of the distance scale for XRF 040912 allows us to investigate the intrinsic properties of this burst. Correcting the peak energy of the $v F_{v}$ prompt emission spectrum for the cosmological redshift, we find $E_{\text {peak }, i}=44 \pm 33 \mathrm{keV}$, while the total isotropic-equivalent released energy implied from the observed fluence during the prompt emission is $E_{\text {iso }}=(1.5 \pm 0.4) \times$ $10^{52} \mathrm{erg}$. These values are consistent with the $E_{\text {peak }, i}-E_{\text {iso }}$ relation found to be valid for a large sample of bursts (e.g. Amati et al. 2002), including GRBs, XRR bursts and XRFs (Fig. 6).

However, the $E_{\text {peak }, i}$ and $E_{\text {iso }}$ values rank XRF 040912 among the intrinsically soft GRBs rather than among the intrinsic XRFs, as evident in Fig. 6. A similar case was reported by Rau et al. (2005) for XRF 030528 at $z=0.782$ that would have been classified as a soft GRB in its rest frame. Two more XRFs with rest frame properties more similar to soft GRBs are XRF 030429 at $z=2.65$ and XRF 981226 at $z=1.11$, although for the latter, the intrinsic peak energy measure is affected by large uncertainty (D'Alessio et al. 2005). In addition, recent studies of XRF 050215B at unknown redshift, have addressed the faintness of its prompt event to a moderate luminosity distance (Levan et al. 2006). Another intermediate case that would have been classified as an X-ray Rich burst is XRF 050416A at $z=0.65$ (Sakamoto et al. 2005) while the only intrinsic XRFs (hereafter $i$-XRFs) detected so far are XRF 020903 at $z=0.2506$ (Sakamoto et al. 2004) and XRF 060218 at $z=0.03342$ (Campana et al. 2006), both with an intrinsic $E_{\text {peak }}$ below $10 \mathrm{keV}^{3}$.

Motivated by these findings, we further investigated the intrinsic properties of other XRFs detected by HETE- 2 with no measured spectroscopic redshift but for which the pseudoredshift was determined using a distance estimator based on the prompt emission (Atteia 2003; Pélangeon et al. 2006). The pseudo-redshifts computed so far have provided redshift estimate within a factor of 2 for tens of bursts (Pélangeon et al. 2006). Its applicability is reinforced here by XRF 040912, with $z_{\mathrm{p}}=2.9 \pm 1.6$ consistent with the spectroscopic redshift of the host galaxy. Two suitable candidates are XRF 021104 and XRF 030823 (Pélangeon et al. 2006) for which the pseudoredshifts are $z_{\mathrm{p}}=1.2 \pm 1.1$ and $z_{\mathrm{p}}=0.8 \pm 0.7$, respectively. For XRF 021104 we find $E_{\text {iso }}=(0.7 \pm 0.2) \times 10^{52} \mathrm{erg}$ and $E_{\text {peak }}=60_{-15}^{+27} \mathrm{keV}$ while for XRF 030823 we find $E_{\text {iso }}=$ $(1.5 \pm 0.2) \times 10^{52} \mathrm{erg}$ and $E_{\text {peak }}=53_{-9}^{+11} \mathrm{keV}$. These values again classify these two bursts among the intrinsic soft GRBs rather than among the $i$-XRFs (Fig. 6, filled triangles). We stress that the pseudo-redshift is an "estimator" of the distance scale and this result cannot be certain, although it is still a plausible explanation.

\section{The nature of X-ray flashes}

These findings point out that the first interpretation of XRFs as high-redshift GRBs is turning out to be correct if XRFs are interpreted as soft GRBs at intermediate redshifts. This is true for all XRFs detected so far, but two. For the two $i$-XRFs 060218 and 020903 another explanation is required ${ }^{3}$. It is worth noting, however, that both GRBs and $i$-XRFs nicely satisfy the $E_{\text {peak }, i}-E_{\text {iso }}$ relation, indicating a possible common origin of the prompt emission mechanism.

We further attempt to extract useful information by comparing the intrinsic properties of XRFs, XRR bursts and GRBs. We compare XRF 040912 with 45 long bursts taken from the burst sample quoted by Amati (2006), for which good estimates of redshift and observed peak energy were obtained. In particular, we consider the $E_{\text {peak }, i}$ value of GRB 031203 as quoted by Amati (2006), although this value is still uncertain (see

3 XRF 040701 at $z=0.215$ may be considered as another intrinsic XRF, with intrinsic $E_{\text {peak }}<6 \mathrm{keV}$ (Amati et al. 2006). 

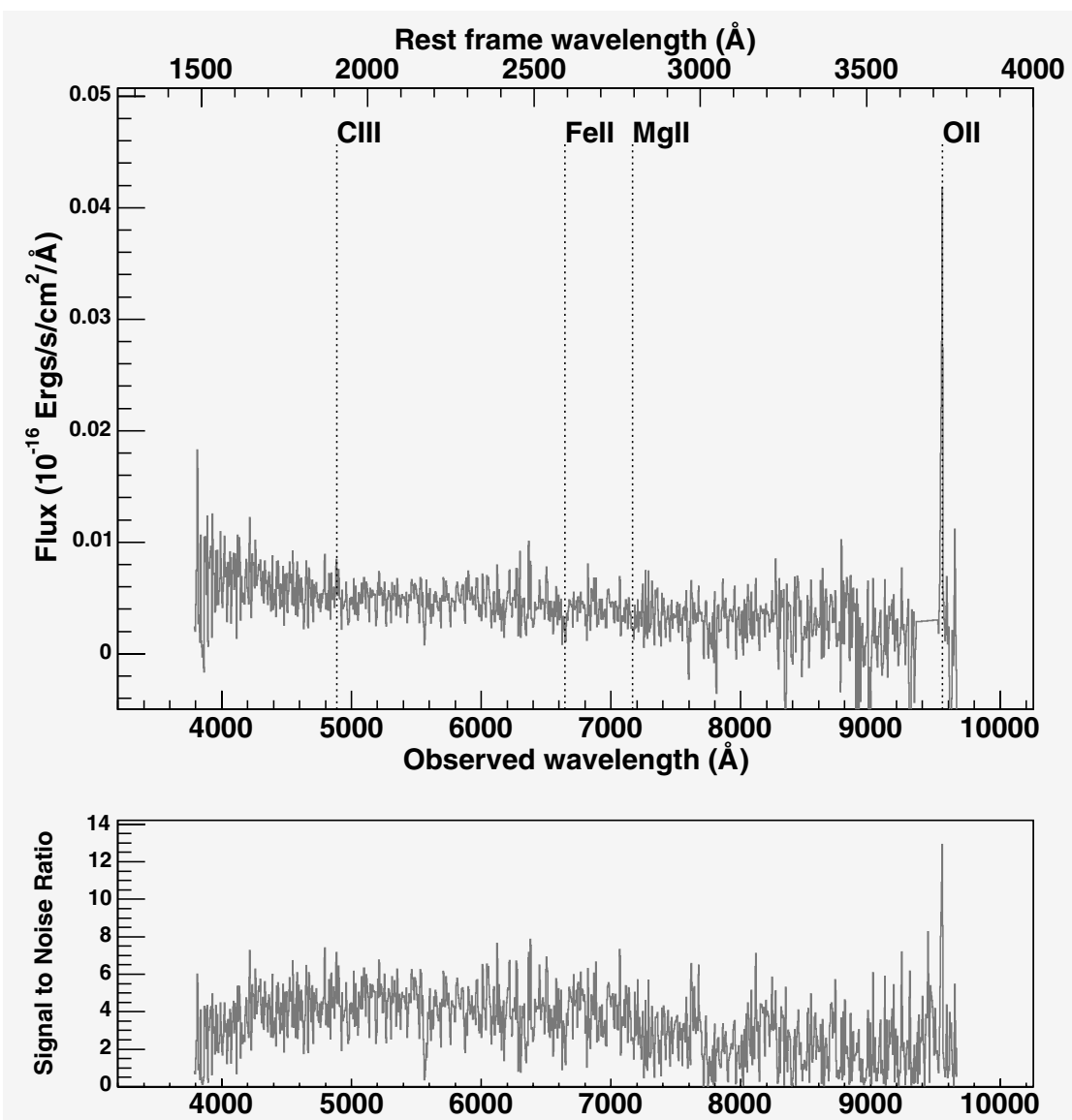

Fig. 3. The likely host galaxy VLT/FORS2 1D spectrum (upper panel) and the associated error spectrum (lower panel). The bad sky subtraction at 9400-9500 ̊ has been hidden. The line identifications imply a redshift $z=1.563 \pm 0.001$.

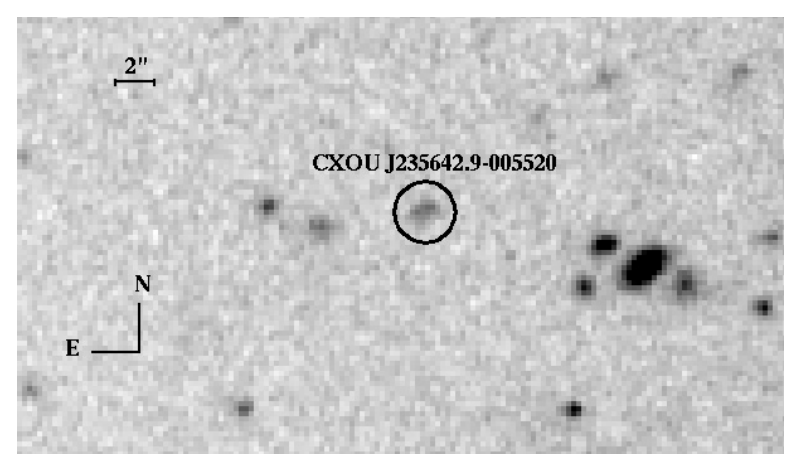

Fig. 4. The $I$-band VLT/FORS2 image of the likely host galaxy of XRF 040912 one year after the burst. The black circle is the Chandra error region of the X-ray afterglow candidate.

also Watson et al. 2006). In Fig. 7 we plot the intrinsic peak energy as a function of redshift. In this plot, the $E_{\text {peak }, i}$ values of XRFs are the natural extension of the $E_{\text {peak }, i}$ of GRBs towards soft values, forming a single population of bursts with $20 \mathrm{keV}<E_{\text {peak }, i}<2000 \mathrm{keV}$, where those bursts with observed peak energy below $40 \mathrm{keV}$ are identified as XRFs. Two possible outliers are the two nearby $i$-XRFs (namely XRF 020903 and XRF 060218). Although at this stage we can not exclude a continuum distribution down to very soft bursts, the gap between the majority of the bursts and these two nearby $i$-XRFs may possibly indicate a different population of very soft bursts (see also Liang et al. 2006). A distinct population of soft and sub-energetic bursts has been recently proposed by Mazzali et al. (2006) where the diversity from the brighter and harder bursts stems from a lower mass progenitor. This scenario was driven not only by the softness and the faintness of the prompt emission of XRF 020903 and XRF 060218, but also by the peculiar properties of their associated $\mathrm{SNe}$ with respect to other GRB-SNe. Despite low mass progenitors (20-25 $\left.M_{\odot}\right)$ possibly being more common than high mass $\left(35-50 M_{\odot}\right)$ ones, the softness of their bursts would make their detection more difficult than for the harder bursts due to the spectral coverage presently afforded by the flying high energy satellites. Similar conclusions have been reached by Liang et al. (2006) from GRB luminosity function studies. The $i$-XRFs segregation is however less evident in the $E_{\text {iso }}$ (or $L_{\text {iso }}$ ) versus redshift diagram (see e.g. Liang et al. 2006). Further identifications of $i$-XRFs will help answer the question of whether the soft peak energy bursts form a distinct population or if they are rather the extension to soft energies of a single phenomenon.

\section{Summary and conclusions}

We performed a multi-wavelength analysis of XRF 040912. We find no optical afterglow counterpart. The limiting magnitude, computed from image subtraction, ranks this burst among the "darkest" ever detected so far, with $R>25.3$ mag at $13.6 \mathrm{~h}$ after the burst. Among $22 \mathrm{X}$-ray sources detected by Chandra in the HETE-2 error-box, only one shows a statistically significant fading behavior with decay rate consistent with the typical values measured for X-ray afterglows at similar epochs (few days after the burst). No evidence of any SN re-brightening has been detected 22.3 days after the burst. The non-fading optical source positionally consistent with the X-ray afterglow candidate, likely 


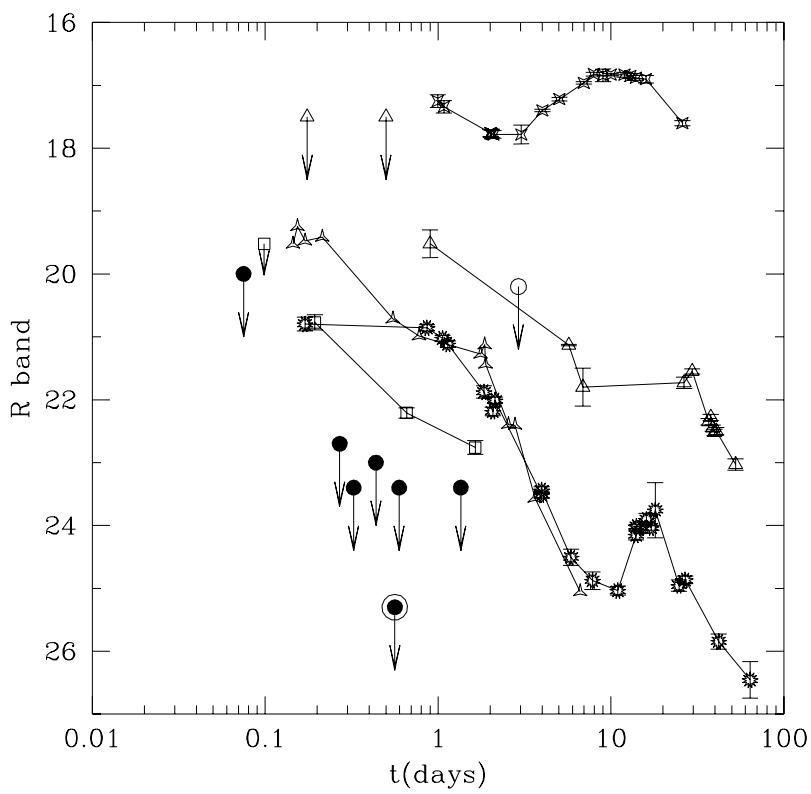

Fig. 5. Optical afterglow $R$-band light curves for the XRFs at known redshift, up to $\sim 50$ days after the burst. The magnitudes were corrected only for Galactic extinction, but for XRF 020903 (upward pointing red triangles, Bersier et al. 2006) and for XRF 060218 (starred open squares) Mirabal et al. (2006c) for which, in addition, the host galaxy subtraction was applied. The other data are from: XRF 030429 (starred open triangles) Jakobsson et al. (2004); XRF 050416A (open squares) Kahharov et al. (2005), Yanagisawa et al. (2005), Holland et al. (2006); XRF 040701, (open circle) de Ugarte Postigo et al. (2004); XRF 030723 (starred open circles) Fynbo et al. (2004). We put for XRF 040912 (filled circles) the deepest optical upper limits for the HETE-2/WXM error-box down to which no variable source was found by the Kiso (Ogura et al. 2004), NOT (Andersen et al. 2004), TLS (this work) and Blanco (Rest et al. 2004) telescopes and the upper limit found at the position of the X-ray afterglow candidate from residual image obtained from Magellan image subtraction (marked filled circle).

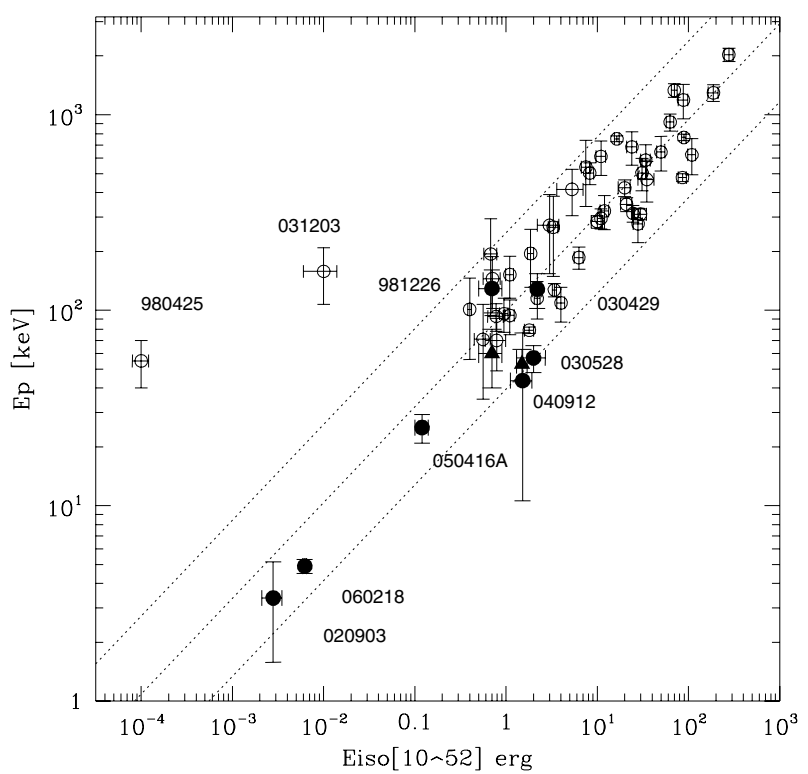

Fig. 6. The $E_{\text {peak }}-E_{\text {iso }}$ relation (Amati et al. 2002; Amati 2006). The open circles are the GRBs and XRR bursts while filled circles are the observed XRFs so far. The dotted lines are the best fit power law $E_{\text {peak }, i}=99 \times E_{\text {iso }}^{0.49}$ delimitated by a logarithmic deviation of 0.4 (Amati 2006). The filled triangles are XRF 021104 and XRF 030823 for which the distance scale has been estimated through the pseudoredshift (Pélangeon et al. 2006).

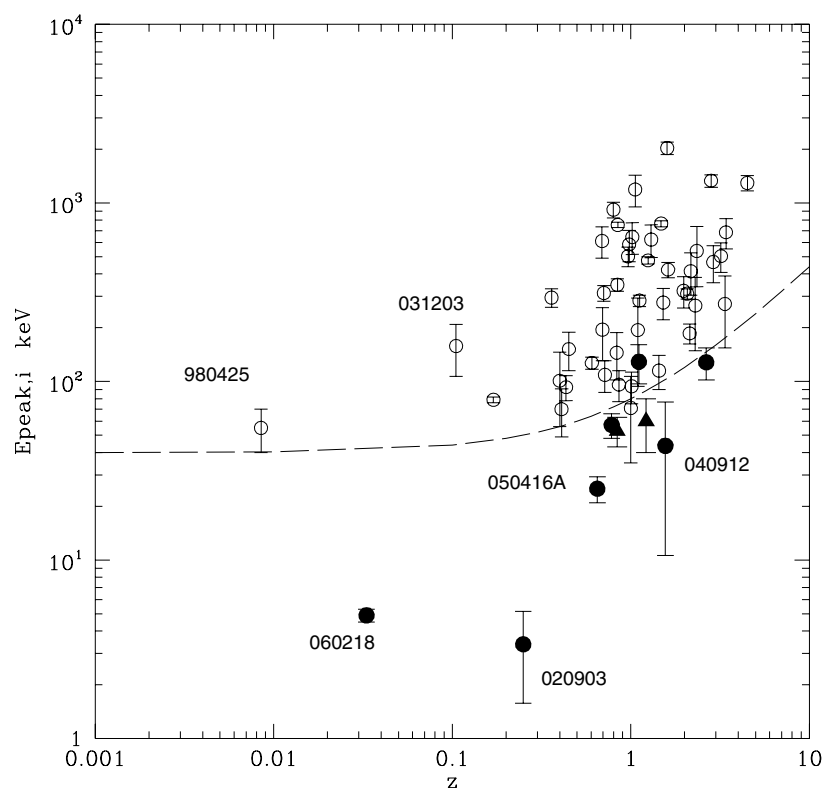

Fig. 7. The intrinsic peak energy $E_{\text {peak }, i}$ of GRBs, XRR bursts (from Amati 2006, open circles) and XRFs (filled circles) is plotted against redshift. The dashed line marks the intrinsic peak energy evolution with redshift, assuming a burst with observed peak energy of $40 \mathrm{keV}$.

the host galaxy, shows a spectrum with a single emission line at $9550 \AA$. The lack of any other strong emission lines blue-ward of the detected one and the absence of the Ly $\alpha$ cut-off down to $3800 \AA$ are consistent with the hypothesis of the [OII] line at redshift $z=1.563 \pm 0.001$. The intrinsic spectral properties rank this XRF among the soft GRBs in the $E_{\text {peak }, i}-E_{\text {iso }}$ diagram. Similar results are obtained for most XRFs at known redshift (or pseudo-redshift), suggesting the existence of two types of XRFs: one type is made by soft GRBs $\left(E_{\text {peak }, i}<40 \mathrm{keV}\right)$ at high $(z \sim 1)$ redshifts (most XRFs are of this type) and another type ( $i$-XRFs), as XRF 060218 and XRF 020903 (see footnote 3), that show intrinsic soft properties possibly associated with a different progenitor population. These observations, may call for a new definition of XRFs, which would be restricted to transients with an $E_{\text {peak }}$ of a few $\mathrm{keV}$. Such a definition is however beyond the scope of this paper.

Acknowledgements. The authors are grateful to Thierry Contini and Jacob Walcher for useful discussion and to the anonymous referee for helpful suggestions. G.S. and B.G. acknowledge the support by the EU Research and Training Network "Gamma-Ray Bursts, an Enigma and a Tool". This work is based in part on observations obtained with MegaPrime/MegaCam, a joint project of CFHT and CEA/DAPNIA, at the Canada-France-Hawaii Telescope (CFHT) which is operated by the National Research Council (NRC) of Canada, the Institut National des Science de l'Univers of the Centre National de la Recherche Scientifique (CNRS) of France, and the University of Hawaii and on observations collected at the European Southern Observatory (ESO), Chile, under Director's Discretionary Time Proposal 275.A-5041.

\section{References}

Alard 2000, A\&A, 144, 363

Amati, L. 2006, MNRAS, 372, 233

Amati, L., Frontera, F., Tavani, M., et al. 2002, A\&A, 390, 81

Amati, L. et al. 2006, A\&A, submitted, [arXiv: astro-ph/0607148]

Andersen, M. I., Weidinger, M., Hjorth, J., Fynbo, J. P. U., \& Jensen, B. L. 2004, GCN, 2707

Atteia, J. L. 2003, A\&A, 407, L1

Band, D. L., Matteson, J., Ford, L., et al. 1993, ApJ, 413, 281

Barraud, C., Olive, J.-F., Lestrade, J. P., et al. 2003, A\&A, 400, 1021 
Barraud, C., Daigne, F., Mochkovitch, R., \& Atteia, J. L. 2005, A\&A, 440, 809 Bersier, D., Fruchter, A. S., Strolger, L.-G., et al. 2006, ApJ, 643, 284 Bertin, E., \& Arnouts, S. 1996, A\&AS, 117, 393

Bruzual, G., \& Charlot, S. 2003, MNRAS, 344, 1000

Butler, N., Ford, P., Ricker, G., et al. 2003, GCN, 2347

Butler, N., Ricker, G., Atteia, J.-L., et al. 2004a, GCN, 2701

Butler, N., Ford, P., Ricker, G., et al. 2004b, GCN, 2716

Butler, N., Ricker, G., Vanderspek, R., et al. 2004c, GCN, 2728

Campana, S., \& Moretti, A. 2004, GCN, 2649

Campana, S., Mangano, V., Blustin, A. J., et al. 2006, Nature, 442, 1008

Chen, S. L., Li, A. G., \& Wei, D. M. 2006, ApJ, 647, L13

Christensen, L., Hjorth, J., \& Gorosabel, J. 2004, A\&A, 425, 913

Christensen, L., Hjorth, J., \& Gorosabel, J. 2005, A\&A,631, L29

Crew, G., Ricker, G., Atteia, J.-L., et al. 2005, GCN, 3890

D’Alessio, V., Piro, L., \& Rossi, E. M. 2005, A\&A, in press [arXiv: astro-ph/0511272]

De Pasquale, M., Piro, L., Gendre, B., et al. 2006, A\&A, 455, 813

Dermer, C. D., Chiang, J., \& Böttcher, M. 1999, ApJ, 513, 656

de Ugarte Postigo, A., Tristram, P., Gorosabel, J., Yock, P., \& Castro-Tirado, A. J. 2004, GCN, 2621

Dickey, J. M., \& Lockman, F. J. 1990, ARA\&A, 28, 215

Ford, P., Butler, N., Ricker, G., et al. 2004, GCN, 2731

Fynbo, J. P. U., Sollerman, J., Hjorth, J., et al. 2004, ApJ, 609, 962

Fynbo, J. P. U., Jensen, B. L., Sollerman, J., et al. 2005, GCN, 3874

Fox, D. B. 2002, GCN, 1392

Fox, D. B. 2004, GCN, 2630

Gorosabel, J., Christensen, L., Hjorth, J., et al. 2003, A\&A, 400, 127

Gorosabel, J., Cepa, J., de Ugarte Postigo, A., et al. 2004, GCN, 2724

Heise, J., in 't Zand, J., Kippen, R. M., \& Woods, P. M. 2001, in Proc. 2nd Rome Workshop: Gamma Ray Bursts in the Afterglow Era, ed. E. Costa, F. Frontera, \& J. Hjorth (Berlin: Springer-Verlag), 16

Heise, J. 2003, Gamma Ray Bursts and the Afterglow Astronomy 2001: A Workshop Celebrating the First Year of the HETE Mission, AIP Conf. Proc. 662,229

Henden, A. 2004, GCN, 2720

Holland, S. T., Boyd, P. T., Gorosabel, J., et al. 2006 [arXiv:astro-ph/0604316]

Jakobsson, P., Hjorth, J., Fynbo, J. P. U., et al. 2004, A\&A, 427, 785

Kahharov, B., Ibrahimov, M., Sharapov, D., et al. 2005, GCN, 3274

Kelson, D. D., Koviak, K., Berger, E., \& Fox, D. B. 2004, GCN, 2627

Kennicutt, R. C. Jr. 1998, ARA\&A, 36, 189

Klose, S., Laux, U., Greiner, J., et al. 2004, GCN, 2708

Kippen, P. 2001, in Gamma Ray Bursts in the Afterglow Era, 22

Lamb, D. Q., Donaghy, T. Q., \& Graziani, C. 2005, ApJ, 620, 355

Liang, E., Zhang, B., Virgili, F., \& Dai, Z. G. 2006, ApJ, submitted [arXiv:astro-ph/0605200]

Levan, A. J., Fruchter, A., Rhoads, J., et al. 2006, ApJ, 647, 471

Maiolino, R., Marconi, A., \& Oliva, E. 2000, A\&A, 365, 37

Mangano, V., La Parola, V., Cusumano, G., et al. 2006, ApJ, in press [arXiv:astro-ph/0603738]

Mazzali, P. A., Deng, J., Nomoto, K., et al. 2006, Nature, 442, 1018

Mészáros, P., Ramirez-Ruiz, E., Rees, M. J., \& Zhang, B. 2002, ApJ, 578, 812

Mirabal, N., Halpern, J. P., An, D., Thorstensen, J. R., \& Terndrup, D. M. 2006, AJ, 643, L99

Mochkovitch, R., et al. 2004, in Third Rome Workshop on Gamma-Ray Bursts in the Afterglow Era, ed. M. Feroci, et al. (San Francisco: ASP), ASP Conf. Ser., 312, 381

Ogura, K., Nishiura, S., Isogai, M., \& Urata, Y., et al. 2004, GCN, 2705

Pélangeon, A., and the Hete-2 Science Team 2006, the 16th Annual October Astrophysics Conference in Maryland, Gamma Ray Bursts in the Swift Era, Washington DC., ed. D. Holt, N. Gehrels, \& J. A. Nousek, November 29-December 2, 836, 1492005

Pian, E., Mazzali, P. A., Masetti, N., et al. 2006, Nature, 442, 1011

Piro, L., Frail, D. A., Gorosabel, J., et al. 2002, ApJ, 577, 680

Rau, A., Salvato, M., \& Greiner, J. 2005, A\&A, 444, 425

Rest, A., Rhoads, J., Weilbacher, P., et al. 2004, GCN, 2710

Rol, E., Wijers, R. A. M. J., Kouveliotou, C., Kaper, L., \& Kaneko, Y. 2005, ApJ, 624,868

Romano, P., Moretti, A., Banat, P. L., et al. 2006, A\&A, 450, 59

Sakamoto, T., Lamb, D. Q., Graziani, C., et al. 2004, ApJ, 602, 875
Sakamoto, T., Kulkarni, S. R., Berger, E., et al. 2005, ApJ, 629, 311 Schlegel, D. J., Finkbeiner, D. P., \& Davis, M. 1998, ApJ, 500, 525 Soderberg, A. M., Kulkarni, S. R., Berger, E., et al. 2004, ApJ, 606, 994 Soderberg, A., Nakar, M. E., Cenko, S. B., et al. 2006, ApJ, submitted [arXiv: astro-ph/0607511]

Stratta, G., Malacrino, F., Boer, M., et al. 2004a, GCN, 2797

Stratta, G., Fiore, F., Antonelli, L. A., Piro, L., \& De Pasquale, M. 2004b, ApJ, 608,846

Watson, D., Vaughan, S. A., Willingale, R., et al. 2006, ApJ, 636, 967

Yamazaki, R., Ioka, K., \& Nakamura, T. 2002, ApJ, 571, L31

Yanagisawa, K., Toda, H., Kawai, N., et al. 2005, GCN, 3287

1 Laboratoire d'Astrophysique de Toulouse, Observatoire MidiPyrénées, 14 Av. E. Belin, 31400 Toulouse, France

e-mail: gstratta@ast.obs-mip.fr

2 Laboratoire d'Astrophysique de Marseille, Traverse du Siphon-Les trois Lucs, 13012 Marseille, France

3 Space Sciences Laboratory, University of California at Berkeley, 445 Campbell Hall, Berkeley, CA 94720, USA

4 INAF/IASF Roma, via fosso del Cavaliere 100, 00133 Roma, Italy

5 Institut d'Astrophysique de Paris, 98bis boulevard Arago, 75014 Paris, France

6 Thüringer Landessternwarte Tautenburg, Sternwarte 5, 07778 Tautenburg, Germany

7 INAF/IASF Bologna, via Gobetti 101, 40129 Bologna, Italy

8 Instituto de Astrofísica de Andalucía (IAA-CSIC), Apartado de Correos 3004, 18080 Granada, Spain

9 Instituto de Astrofísica de Canarias (IAC), C. via Láctea s/n, La Laguna, 38200 Tenerife, Spain

10 Observatoire Haute Provence (CNRS/OAMP), Saint Michel l'Observatoire, France

11 Instituto Nacional de Pesquisas Espaciais, Avenida Dos Astronautas 1758, São José dos Campos 12227-010, Brazil

12 Center for Space Research, MIT, 70 Vassar Street, Cambridge, MA 02139, USA

13 Department of Astronomy and Astrophysics, University of Chicago, 5640 South Ellis Avenue, Chicago, IL 60637, USA

14 Centre d'Étude Spatiale des Rayonnements, Observatoire MidiPyrénées, 9 avenue de Colonel Roche, 31028 Toulouse, France

15 Los Alamos National Laboratory, PO Box 1663, Los Alamos, NM, 87545, USA

16 University of California at Berkeley, Space Sciences Laboratory, Berkeley, CA, 94720-7450, USA

17 Department of Physics, Tokyo Institute of Technology, 2-12-1 Ookayama, Meguro-ku, Tokyo 152-8551, Japan

18 RIKEN (Institute of Physical and Chemical Research), 2-1 Hirosawa, Wako, Saitama 351-0198, Japan

19 Department of Astronomy and Astrophysics, Tata Institute of Fundamental Research, Homi Bhabha Road, Mumbai, 400 005, India

20 Tsukuba Space Center, National Space Development Agency of Japan, Tsukuba, Ibaraki 305-8505, Japan

21 National Astronomical Observatory, 2-21-1, Osawa, Mitaka, Tokyo 181-8588, Japan

22 Department of Physics, Aoyama Gakuin University, Chitosedai 616-1 Setagaya-ku, Tokyo 157-8572, Japan

23 Faculty of engineering, Miyazaki University, Gakuen Kibanadai Nishi, Miyazaki 889-2192, Japan

24 Department of Astronomy and Astrophysics, University of California at Santa Cruz, 477 Clark Kerr Hall, Santa Cruz, CA95064, USA

25 NASA Goddard Space Flight Center, Greenbelt, MD, 20771, USA 